\title{
CARDIOTOCOGRAPHY DURING LABOUR AND ITS RELATION WITH NEONATAL OUTCOME
}

Verma Aruna, Gupta Abhilasha

1. Assistant Professor. Department of Obstetrics \& Gynaecology, L. L. R. M Medical College, Meerut, Uttar Pradesh.

2. Professor \& HOD. Department of Obstetrics \& Gynaecology, L. L. R. M Medical College, Meerut, Uttar Pradesh.

\section{CORRESPONDING AUTHOR}

Dr. Aruna Verma,

Assistant Professor,

Department of Obstetrics \& Gynaecology,

L.L.R.M. Medical College, Meerut (U.P.), India.

E-mail: arunatonk@yahoo.co.in

Ph: 00919997706487

CAPSULE: 120 cases of high risk as well as low risk pregnancy at term in labor were admitted in labor room of S.V.B.P Hospital (attached to L.L.R.M Medical College, Meerut). Continuous E.F.H.R.M was done by Hewlett Packard Cardio-tocograph and findings were correlated with neonatal outcome.

KEY WORDS: Cardiotocography, Electronic fetal heart rate monitoring, Fetal heart rate patterns

ABSTRACT: Monitoring of health of fetus during labor is critical component of obstetric care. Present study was done to determine the clinical usefulness of continuous electronic fetal heart rate monitoring for identification of distressed fetus.

The present study was conducted on 120 cases who were admitted in labor room during labor in SVBP hospital MRT. Continuous EFHRM was done by Hewlett Packard Cardiotocograph and findings were correlated with neonatal outcome.

Cases with high risk factors(70), who had reassuring patterns (i.e. 44 cases) ,all were delivered vaginally but with non reassuring patterns only $8(30.8 \%)$ were delivered normally $.84(70 \%)$ cases with reassuring patterns had zero neonatal mortality .But 36(30\%) with nonreassuring patterns 3 neonates expired in spite of all resuscitative measures. But out of 84 reassuring FHR patterns, 10 neonates were diseased i.e they required NICU admission.

Continuous EFHRM detected hypoxia even before asphyxia developed and had sensitivity of $84.1 \%$ and specificity of $68.75 \%$.False negatives were $15.9 \%$,false positives were $31.25 \%$.Predictive values of reassuring and non-reassuring patterns were $88 \%$ \& $61 \%$ respectively.

INTRODUCTION: During the past two decades, remarkable intimate knowledge of human fetus along with technological development have prompted a new phenomena in medicine forecast of fetal health. Fetal evaluation in the intra-partum period is increasing to improve perinatal morbidity and mortality rates. Intra-partum fetal surveillance is aimed to detect the fetus in the process of deterioration during labor so that appropriate intervention can be undertaken to prevent fetal jeopardy as earliest as possible. Commencement of such monitoring, soon after admission in early labor, is designed to detect the earliest sign of fetal distress using the principle that early uterine contractions act as spontaneous stress test and changes in fetal 
heart rate patterns with this stress help in the selection of cases for intensive intra-partum fetal monitoring in low \& high risk group. By the start of $20^{\text {th }}$ century, auscultation of fetal heart rate by stethoscope had become established as a means of evaluating fetal health. The desire for better tool led to development of EFHRM (by cardio-tocographic machine).Before CTG monitoring, it is important to identify the mother who is generally at risk for development of intra-partum hypoxia. Unfortunately intra-partum fetal morbidity \& mortality are not uncommon in a low risk population \& it is estimated that up to $20 \%$ low risk pregnancies also become high risk in labor, some of them being probably missed as high risk pregnancies.

External (indirect) EFHRM has gained popularity because of easy application of the equipment, documentation of FHR. It is non invasive \& simultaneously records FHR patterns, fetal movements, uterine contractions \& can discriminate between the fetus at risk for hypoxic injury and the normally oxygenated fetus.

MATERIAL \& METHOD: The present prospective observational study was conducted in the Obs \& Gynae deptt. of a teaching hospital in western U.P , from November 2002 to September 2003. 120 cases were randomly allocated for the study at admission in early labor. The study population consisted of 50 case of low or no risk \& 70 cases of high risk pregnancy. The high risk pregnancies included-

- Pregnancy induced hypertension with or without proteinuria.

- Preterm labor(>30 wks of gestation)

- Previous one caesarian section.

- IUGR.

- Pregnancy with diabetes.

- Pregnancy with anemia.

All cases who did not deliver within $24 \mathrm{hrs}$ or delivered too rapidly, fetus with known congenital anomaly, cases for elective Caesarian \& gestation $<30 \mathrm{wks}$, were excluded from the study. All cases were subjected to EFM for 20 minutes using electronic CTG machine at admission in labor \& then continuous CTG when lady reached in active labor. The tracings thus obtained were classified as reassuring \& non-reassuring fetal heart rate patterns. The following fetal indicators of intra-partum hypoxia were recorded in present study-

- Abnormal fetal heart rate patterns as detected by EFM

- Meconium stained liquor

- Apgar score at 5 minute

- Neonates required NICU admission for intensive neonatal care

- Perinatal mortality due to fetal hypoxia

Statistical analysis of the observations \& the results of above study were carried out using chisquare test in order to find out if the results of study were statistically significant

OBSERVATIONS \& RESULTS: In the present study it was observed that a higher proportion of normal pregnancies $(80 \%)$ had reassuring fetal heart rate patterns as compared to $63 \%$ in high risk group (Table-1).It was, however interesting to note that $20 \%$ of so called normal / low risk pregnancies showed non-reassuring FHR patterns \& thus became high risk in labor (Table- 
2).Out of total high risk cases(70),37\% had meconium staining at rupture of membranes \& out of 26 cases of meconium staining only 4 cases i.e. $15 \%$ were found to be having reassuring FHR patterns (Table-3).

Cases with high risk factors(70), who had reassuring patterns (i.e. 44 cases) ,all were delivered vaginally but with non reassuring patterns only $8(30.8 \%)$ were delivered normally (Table-4).All cases with reassuring pattern had mean apgar of 8 but 10(12\%) babies needed NICU admission later on due to some other reasons but no mortality noted. With nonreassuring patterns $94.4 \%$ neonates had mean apgar score $<7$ at $5 \mathrm{~min}, 60 \%$ required NICU admission(Table-5).

$84(70 \%)$ cases with reassuring patterns had zero neonatal mortality .But 36(30\%) with non- reassuring patterns 3 neonates expired in spite of all resuscitative measures (Table-6). But out of 84 reassuring FHR patterns, 10 neonates were diseased i.e they required NICU admission.

DISCUSSION: Intra-partum fetal surveillance using EFM has gained increasing importance in recent years in our quest to improve perinatal morbidity \& mortality (1). The Cochrane review evaluated the effectiveness \& safety of CTG (2) It is presumed to be superior method for fetal hypoxia as it detects subtle changes in fetal heart rate which can be missed on auscultation. In the present prospective study the various FHR patterns i.e. no risk as well as high risk pregnancies \& their correlation with perinatal outcome( including mode of delivery ,apgar score, NICU admission, neonatal deaths) were studied. In present study caesarian rates were very high in non reassuring/ pathological patterns but overall incidence of caesarian were less (23.3) as similar to INGEMARSSON' STUDY $(3,4)$.

Specific decelerative patterns were definitely associated with disturbances in acid base balance; this is true specially of late \& severe variable deceleration. The corresponding mean apgar for late \& variable deceleration were $5.4 \& 5.9$ respectively (5) comparable to our study (5.8 \& 6.8 respectively). Presence of late deceleration is ominous characteristic associated with a significant increase in perinatal morbidity \& mortality(6).Same is also found in our study that most neonatal deaths occurred in late decelerative patterns. Delinger et al have demonstrated that intra-partum FHR patterns correlate well with fetal academia \& umbilical blood gases (7). Sameshima et al (8) have shown these ominous patterns to be consistently associated with increased incidence of fetal distress, fetal academia, resulting in caesarian delivery or admission to NICU. Decreased $(<5 \mathrm{bpm})$ or absent variability along with frequent severe variable or late deceleration is constantly associated with fetal hypoxia \& acidemia. Such extreme forms of fetal distress are very rare, which explains why the benefits derived from continuous FHRM are not very convincing (9), as also found in our study. Ahn et al have shown that there is no unique FHR patterns which is associated with brain damage in the fetus(10).Schiermeier et al (11) showed a high sensitivity(95\%) for FIGO classification 'suspect' \& 'pathological', together with a low specificity(21.8) for fetal acidosis. Ingemarsson (1986) found high predictive value of normal FHR patterns (98.7\%) but rather a low predictive value of abnormal FHR patterns (12). Both studies are comparable to our study.

Thus following have been suggested as additional means to reduce the false positive predictions of fetal distress compared to when EFM is used alone (A)-Fetal scalp blood ph. (B) Fetal pulse oximetery. (C) Fetal scalp stimulation \& VAS, (D) Fetal ECG.

CONCLUSION: Thus with careful interpretation various FHR patterns can be useful as screening test for fetal hypoxia /asphyxia .But because of high sensitivity, low specificity \& a large no. of 
false positive patterns, various supplementary tests like scalp sampling, pulse oximetry are necessary to confirm the diagnosis and to avoid unnecessary intervention.

\section{REFERENCES:}

1. Smith JH-Obstetrics in the 1990s:Current controversies. Oxford:Blackwell Scientific, 192-201, 1992.

2. Alfirevic Z, Devane D et al. CTG as a form of electronic fetal monitoring for fetal assessment during labour.(Cochrane Review).The Cochrane Database of Systematic Reviews. Issue 3.2006,Chichester,UK: John Wiley \& sons

3. Ingemarsson E, Ingemarsson I, Solum T, Westgren M: A one year study of routine fetal heart rate monitoring during first stage of labour. Acta Obstet Gynecol Scand 59: 297300,1980

4. Ingemarsson E, Arulkumaran et al:Admission test : a screening test for fetal distress in labor. Obstet Gynecol 68:800-6, 1986.

5. Bisonette JM :Relationship between continuous fetal heart rate patterns \& apgar score in newborn.Br. J.Obstet. Gynecol.82-84,1975.

6. Kulkarni A: Admission test: A predictive test for fetal distress in high risk labour.J.Obstet.Gynecol.Res.24;255-9; 1998.

7. Delinger EH, Boehm FH, Crane M M. Electronic Fetal Heart Rate Monitoring: Early neonatal outcome associated with normal rate, fetal stress \& fetal distress. Am J Obstet Gynecol2000:182:214-98

8. Sameshima H, Ikenoue et al. Unselected low risk pregnancies \& the effect of continuous intrapartum FHRM on umbilical blood gases \& cerebral palsy. Am J Obstet Gynecol 2004;190: 118-23

9. Horbuckle J, Vail A et al. The example of intrapartum electronic FHRM. BJOG 2000:107:3-7.

10. Ahn MO et al. Intrapartum fetal heart rate patterns in 209 brain damaged infants. Am $\mathrm{j}$ Obstet Gynecol 1996;174;492-5

11. Schiermeir $S$ et al.Sensitivity \& specificity of intrapartum computerized FIGO criteria for cardiotocography \& fetal scalp ph during labour:multicenter, observational study. BJOG2008: Vol.115, Issue 12,1557-63.

12. Ingemarrsson I et al.Admission test: a screening test for fetal distress in labor.Obstet Gynecol 68:800-6, 1986. 
Table-I Incidence of Reassuring Patterns

\begin{tabular}{|l|l|l|l|l|l|}
\hline \multirow{2}{*}{ S. No. } & \multirow{2}{*}{ Cases } & \multicolumn{2}{l|}{ Total } & \multicolumn{2}{l|}{ Reassuring Pattern } \\
\cline { 3 - 6 } & & No. & $\mathbf{\%}$ & No. & $\mathbf{\%}$ \\
\hline 1. & No risk Factor & 50 & 42.0 & 40 & 80.0 \\
\hline 2. & High risk Factor & 70 & 58.0 & 44 & 63.0 \\
\hline & (a) PIH & 4 & & 4 & 6.0 \\
\hline & (b) Preeclampsia & 8 & & 6 & 8.5 \\
\hline & (c) Anemia & 8 & & 6 & 8.5 \\
\hline & (d) Preterm & 8 & & 4 & 5.7 \\
\hline & (e) Post dated & 10 & & 2 & 2.9 \\
\hline & (f) IUGR & 4 & & 2 & 2.9 \\
\hline & (g) Rh negative & 8 & & 4 & 5.7 \\
\hline & (h)Previous one LSCS & 14 & & 12 & 17.1 \\
\hline & (i)Diabetes & 6 & & 2 & 5.1 \\
\hline & Total & $\mathbf{1 2 0}$ & & $\mathbf{8 4}$ & \\
\hline
\end{tabular}

Table-II Incidence of Nonreassuring Patterns

\begin{tabular}{|l|l|l|l|l|l|}
\hline \multirow{2}{*}{ S. No. } & \multirow{2}{*}{ Cases } & \multicolumn{2}{|l|}{ Total } & \multicolumn{2}{l|}{ Nonreassuring Pattern } \\
\cline { 3 - 6 } & & No. & $\mathbf{\%}$ & No. & $\mathbf{\%}$ \\
\hline 1. & No risk Factor & 50 & 42.0 & 10 & 20.0 \\
\hline 2. & High risk Factor & 70 & 58.0 & 26 & 37.0 \\
\hline & Total & $\mathbf{1 2 0}$ & $\mathbf{1 0 0 . 0}$ & $\mathbf{3 6}$ & \\
\hline
\end{tabular}

Table-III Reassuring And Non-Reassuring Patterns In Relation To Meconium Stained Liquor

\begin{tabular}{|c|c|c|c|c|c|c|}
\hline \multirow[t]{2}{*}{ Fetal Rate Patterns } & \multicolumn{2}{|c|}{$\begin{array}{l}\text { Meconium stained with } \\
\text { high risk }\end{array}$} & \multicolumn{2}{|c|}{$\begin{array}{l}\text { Clear liquor with } \\
\text { high risk }\end{array}$} & \multicolumn{2}{|c|}{ Total } \\
\hline & No. & $\%$ & No. & $\%$ & No. & $\%$ \\
\hline 1. Reassuring & 4 & 5.7 & 36 & 51.4 & 40 & 57.1 \\
\hline 2. Non-reassuring & 22 & 31.0 & 8 & 11.4 & 30 & 43 \\
\hline a) Late deceleration & 12 & 17.1 & 4 & 5.7 & 16 & 22.8 \\
\hline b) Variable & 10 & 14.0 & 2 & 3 & 12 & 17 \\
\hline c) Bradycardia & - & - & 2 & 3 & 2 & 3 \\
\hline Total & 26 & & 44 & & 70 & \\
\hline
\end{tabular}

Table-IV Mode of Delivery in Various Patterns in Cases With Risk Factor

\begin{tabular}{|l|l|l|l|l|l|l|}
\hline S.No. & \multirow{2}{*}{ FHR Patterns } & \multirow{2}{*}{ No. } & \multicolumn{2}{|l|}{ Vaginal Delivery } & \multicolumn{2}{l|}{ Cesarean } \\
\cline { 4 - 8 } & & & No. & $\mathbf{\%}$ & No. & $\mathbf{\%}$ \\
\hline 1. & Reassuring & 44 & 44 & 63.0 & - & - \\
\hline 2. & Non-reassuring & 26 & 8 & 30.8 & - & 69.2 \\
\hline & (a) Late Deceleration & 12 & 4 & 15.4 & 8 & 30.8 \\
\hline & (b) Variable deceleration & 12 & 2 & 7.7 & 10 & 38.4 \\
\hline & (c) Fetal bradycardia & 2 & 2 & 7.7 & - & - \\
\hline & Total & $\mathbf{7 0}$ & - & - & $\mathbf{1 8}$ & \\
\hline
\end{tabular}


Table-V Mean Apgar Score In Relation To Various Patterns

\begin{tabular}{|l|l|l|l|}
\hline S.No. & FHR Pattern & Total Cases & Mean Apgar Score at 5 min. \\
\hline I. & Reassuring & 84 & 8.0 \\
\hline II. & Non-reassuring & 36 & \\
\hline & (a) Late Deceleration & 16 & 5.8 \\
\hline & (b) Variable deceleration & 18 & 6.8 \\
\hline & (c) Fetal bradycardia & 02 & 8.0 \\
\hline
\end{tabular}

Table-VI Neonates Required NICU Admission

\begin{tabular}{|l|l|l|l|l|l|l|l|}
\hline \multirow{2}{*}{ S.No. } & FHR Patterns & \multicolumn{2}{|l|}{ No NICU Admission } & \multicolumn{2}{l|}{ NICU Admission } & \multicolumn{2}{l|}{ Total } \\
\cline { 3 - 8 } & & No. & $\%$ & No. & $\%$ & No & $\%$ \\
\hline 1. & Reassuring & 74 & 88.0 & 10 & 12 & 84 & 70 \\
\hline 2. & Non-reassuring (Expired) & 14 & 40.0 & $223(13.6 \%)$ & 60.0 & 36 & 30 \\
\hline \multicolumn{2}{|c|}{ Total } & $\mathbf{8 8}$ & & $\mathbf{3 2}$ & & $\mathbf{1 2 0}$ & \\
\hline
\end{tabular}

